\title{
Effect of Atrial Fibrillation on the Incidence and Outcome of Osteoporotic Fracture

\author{
- A Nationwide Population-Based Study -
}

\author{
Daehoon Kim, MD; Pil-Sung Yang, MD; Tae-Hoon Kim, MD; \\ Jae-Sun Uhm, MD, PhD; Junbeom Park, MD, PhD; Hui-Nam Pak, MD, PhD; \\ Moon-Hyoung Lee, MD, PhD; Boyoung Joung, MD, PhD
}

\begin{abstract}
Background: Both atrial fibrillation (AF) and osteoporosis are common in older adults. The purpose of this study was to investigate whether comorbid AF in patients with osteoporosis is associated with fracture incidence, or death after fracture.

Methods and Results: From the National Health Insurance Service database of Korea, we selected 31,778 patients with osteoporosis. During a median follow-up of 48 months, the incidence of bone fractures was higher in AF patients than in non-AF patients (3.20 vs. 2.18 per 100 person-years), respectively. In the multivariate Cox regression analysis, AF was associated with fracture independently of other risk factors with an adjusted hazard ratio $(\mathrm{HR})$ of 1.21 (95\% confidence interval $[\mathrm{Cl}], 1.02-1.41 ; \mathrm{P}=0.031)$. The mortality rate after fracture was significantly higher in AF patients than it was in non-AF patients (adjusted $\mathrm{HR}, 1.92 ; 95 \% \mathrm{Cl}, 1.35-3.27 ; \mathrm{P}=0.016$ ). After propensity score-matching, AF was consistently associated with a higher risk of osteoporotic fracture and subsequent death after fracture. In AF patients, older age, female sex, being underweight (body mass index $<18.5 \mathrm{~kg} / \mathrm{m}^{2}$ ), decreased physical activity (exercise $<3$ times/week), history of stroke or transient ischemic attack, thiazide use, sedative use, and higher $\mathrm{CHADS}_{2}$ ( $\geq 2$ points) or $\mathrm{CHA}_{2} \mathrm{DS}_{2}$-VASc ( $\geq 2$ points) scores were associated with the incidence of fractures.
\end{abstract}

Conclusions: Comorbid AF in patients with osteoporosis was associated with an increased risk of bone fracture and death after fracture.

Key Words: Atrial fibrillation; Death; Fractures; General population; Osteoporosis

A trial fibrillation (AF) is the most common sustained cardiac arrhythmia in the elderly and it is associated with an increased risk of stroke, heart failure (HF), dementia, and death., ${ }^{\mathbf{1 , 2}}$ Osteoporosis is another common medical issue in the elderly. The 2 conditions share common etiological factors, including age, hypertension (HTN), diabetes mellitus (DM), and HF., ${ }^{\mathbf{3} 4}$ Bone fractures are a frequent and important cause of disability and medical costs worldwide..$^{5,6}$ Prior studies have found that cardiovascular diseases, such as ischemic heart disease, stroke, HF, and peripheral vascular disease, increase a patient's risk of hip and other bone fractures. ${ }^{7-9}$

$\mathrm{AF}$ is a potential risk factor for fractures, because it not only causes structural and functional alterations in the cardiovascular system, but also exerts thromboembolic effects on the nervous system, and disturbs postural steadiness. Therefore, AF is now recognized as an independent risk factor for non-accidental falls. ${ }^{10}$ Vitamin $\mathrm{K}$ antagonists, which are used to prevent stroke in AF, might be associated with reduced bone mineral density (BMD) and increased risk of osteoporotic fracture. ${ }^{11-13}$ With respect to bone strength, the thromboembolic effects of AF may reduce bone mass through reduction in the osseous blood supply. ${ }^{\mathbf{1 4}}$ We thus hypothesized that comorbid AF would be associated with incidental bone fractures. However, there is controversy regarding the association between AF and fracture risk. ${ }^{15-17} \mathrm{We}$ also hypothesized that AF would be related to increased mortality after fracture events. We aimed to investigate whether comorbid AF was associated with fracture occurrence or death in patients with osteoporosis using a large-scale database maintained by the Korean National Health Insurance Service (NHIS).

Received October 23, 2017; revised manuscript received April 4, 2018; accepted April 11, 2018; released online May 24, 2018 Time for primary review: 63 days

Division of Cardiology, Yonsei University College of Medicine, Seoul (D.K., P.-S.Y., T.-H.K., J.-S.U., H.-N.P., M.-H.L., B.J.); Department of Cardiology, CHA Bundang Medical Center, CHA University, Seongnam (P.-S.Y.); and Department of Cardiology, School of Medicine, Ewha Womans University, Seoul (J.P.), Republic of Korea

The first two authors contributed equally to this work (D.K., P.-S.Y.).

Mailing address: Boyoung Joung, MD, PhD, Professor of Medicine, Cardiology Division, Yonsei University College of Medicine, 50 Yonsei-ro, Seodaemun-gu, Seoul 03722, Republic of Korea. E-mail: cby6908@yuhs.ac

ISSN-1346-9843 All rights are reserved to the Japanese Circulation Society. For permissions, please e-mail: cj@j-circ.or.jp 


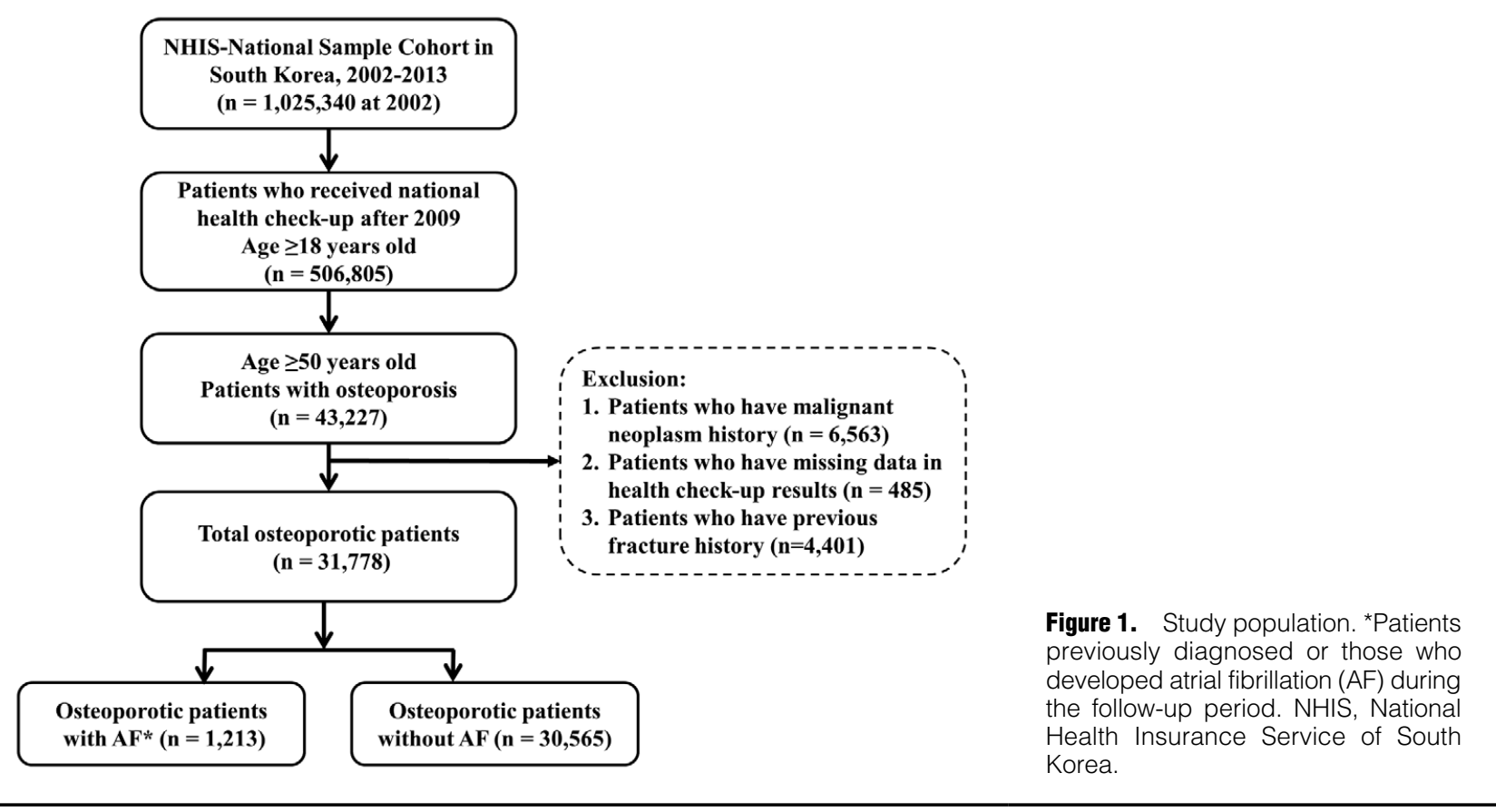

\section{Methods}

\section{Data Source}

We used the National Sample Cohort (NSC) of the NHIS database, which was released by the Korean NHIS in 2015 for research purposes. The Korean NHIS was founded in 1963 as the single insurer managed by the Korean government. Most (97.1\%) South Koreans are mandatory subscribers, with the remaining 3\% of the population being medical aid subjects, so every South Korean is enrolled in the NHIS database. The NHIS-NSC database consists of detailed healthcare data from 1,025,340 enrollees, representing approximately $2.2 \%$ of Korea's population in $2002(46,604,433)$. These patients were followed for 11 years (up to 2013). The database contains the following medical information: sociodemographic information, use of inpatient and outpatient services, pharmacy dispensing claims, and mortality data. Because all enrollees are required to undergo at least biennial national health examinations, the database also provides baseline laboratory data and health-related risk factors, which were updated periodically during the 5-year followup period (January 2009-December 2013). ${ }^{18}$

This study was approved by the Institutional Review Board of Yonsei University Hospital. Informed consent was waived. Data were fully de-identified prior to analysis.

\section{Study Population and Data Collection}

Among 506,805 subjects (aged $\geq 18$ years) who underwent national health examinations between 2009 and 2013, a total of 43,227 patients aged $\geq 50$ years with a diagnosis of osteoporosis during the screening period from January 2002 to December 2008 were identified. Patients with malignant neoplasms $(n=6,563)$ were excluded in order to avoid pathologic fractures related to underlying malignancies. A total of 485 patients were excluded because of missing health examination data. Patients with a history of bone fracture $(n=4,401)$ were excluded. Finally, 31,778 patients were included (Figure 1).

Baseline comorbidities were evaluated during the 7-year screening period (January 2002-December 2008), and identified from the medical claims according to the codes of the International Classification of Disease 10th Revision (ICD-10). Osteoporosis was diagnosed using ICD-10 codes M80-, M81-, and M82- (excluding M82.0; osteoporosis in multiple myelomatosis). AF was diagnosed using ICD-10 code I48, which includes both $\mathrm{AF}$ and atrial flutter. We excluded patients with a record of either mitral stenosis (I05.0, I05.2, and I34.2) or prosthetic heart valves (Z95.2Z95.4), resulting in a typical "non-valvular" AF population. In order to ensure diagnostic accuracy, we defined patients with osteoporosis, AF, and other comorbidities (including HF, HTN, DM, chronic obstructive pulmonary disease [COPD], chronic kidney disease [CKD], peripheral arterial disease [PAD], a history of myocardial infarction [MI], and a history of stroke and/or transient ischemic attack [TIA]) when it was a discharge diagnosis or was confirmed at least twice in an outpatient setting, which was similar to previous studies using the NHIS-NSC.19-21 The definitions of comorbidities are presented in Table S1. The CHADS 2 and $\mathrm{CHA}_{2} \mathrm{DS}_{2}$-VASc scores for each subject were estimated at the end of the screening period. Prescription medication use was ascertained by identifying NHIS database claims. To avoid underestimation of aspirin utilization because of over-the-counter (OTC) purchases in the study population, we included this source in our analysis.

\section{Outcomes and Follow-up}

The primary clinical outcome was a bone fracture requiring hospitalization during the 5-year follow-up period (January 2009-December 2013). Bone fracture was based on the principal diagnosis of hospital admission according to the ICD-10 codes (Table S1). To exclude fractures caused by trauma, any fractures that accompanied a record of motor 


\begin{tabular}{|c|c|c|c|}
\hline \multirow{2}{*}{ Characteristics } & \multicolumn{3}{|c|}{ All patients with osteoporosis $(n=31,778)$} \\
\hline & AF $(n=1,213)$ & Non-AF $(n=30,565)$ & $P$ value \\
\hline Age, years & $69.9 \pm 8.4$ & $65.3 \pm 8.7$ & $<0.001$ \\
\hline Female & $1,031(85.0)$ & $28,086(91.9)$ & $<0.001$ \\
\hline BMI, $\mathbf{k g} / \mathrm{m}^{2}$ & $24.4 \pm 3.6$ & $24.1 \pm 3.2$ & 0.003 \\
\hline Ex-/current smoker & $137(11.3)$ & $2,463(8.1)$ & $<0.001$ \\
\hline Alcohol use, glasses/week & $1.5 \pm 6.4$ & $1.8 \pm 8.3$ & 0.167 \\
\hline Physical activity, times of exercising/week ${ }^{*}$ & $2.6 \pm 2.6$ & $2.8 \pm 2.6$ & 0.009 \\
\hline CHADS $_{2}$ score & $2.1 \pm 1.4$ & $1.1 \pm 1.2$ & $<0.001$ \\
\hline $\mathrm{CHA}_{2} \mathrm{DS}_{2}$-VASc score & $3.9 \pm 1.7$ & $2.7 \pm 1.6$ & $<0.001$ \\
\hline \multicolumn{4}{|l|}{ Medical history } \\
\hline HF & $404(33.3)$ & $2,289(7.5)$ & $<0.001$ \\
\hline HTN & $983(81.0)$ & $16,440(53.8)$ & $<0.001$ \\
\hline DM & $271(22.3)$ & $4,379(14.3)$ & $<0.001$ \\
\hline History of stroke/TIA & $291(24.0)$ & $3,689(12.1)$ & $<0.001$ \\
\hline History of MI & $100(8.2)$ & $599(2.0)$ & $<0.001$ \\
\hline PAD & $394(32.5)$ & $7,259(23.7)$ & $<0.001$ \\
\hline COPD & $335(27.6)$ & $4,878(16.0)$ & $<0.001$ \\
\hline CKD & $351(28.9)$ & $4,360(14.3)$ & $<0.001$ \\
\hline \multicolumn{4}{|l|}{ Cardiac medications } \\
\hline Antiplatelet drugs & $677(55.8)$ & $8,462(27.7)$ & $<0.001$ \\
\hline Vitamin $\mathrm{K}$ antagonists & $288(23.7)$ & $97(0.3)$ & $<0.001$ \\
\hline$\beta$-blockers & $538(44.4)$ & $6,030(19.7)$ & $<0.001$ \\
\hline Calcium-channel blockers & $618(50.9)$ & $10,363(33.9)$ & $<0.001$ \\
\hline Digoxin & $210(17.3)$ & $261(0.9)$ & $<0.001$ \\
\hline Antiarrhythmic drugs* & $100(8.2)$ & $47(0.2)$ & $<0.001$ \\
\hline ACEI or ARB & $597(49.2)$ & $7,801(25.5)$ & $<0.001$ \\
\hline Loop diuretics & $210(17.3)$ & $943(3.1)$ & $<0.001$ \\
\hline Thiazide diuretics & $427(35.2)$ & $6,084(19.9)$ & $<0.001$ \\
\hline Spironolactone & $161(13.3)$ & $856(2.8)$ & $<0.001$ \\
\hline HMG-CoA reductase inhibitors & $457(37.7)$ & $7,877(25.8)$ & $<0.001$ \\
\hline \multicolumn{4}{|l|}{ Noncardiac medications } \\
\hline Proton pump inhibitors & $169(13.9)$ & $2,948(9.6)$ & $<0.001$ \\
\hline Corticosteroids & $149(12.3)$ & $3,615(11.8)$ & 0.630 \\
\hline Osteoporosis medications ${ }^{\dagger}$ & $182(15.0)$ & $5,561(18.2)$ & 0.005 \\
\hline Thyroid replacement & $66(5.4)$ & $1,272(4.2)$ & 0.030 \\
\hline Anticonvulsants & $146(12.0)$ & $2,491(8.1)$ & $<0.001$ \\
\hline Sedatives ${ }^{\ddagger}$ & $618(50.9)$ & $11,170(36.5)$ & $<0.001$ \\
\hline Selective serotonin reuptake inhibitors & $41(3.4)$ & $571(1.9)$ & $<0.001$ \\
\hline
\end{tabular}

Data are expressed mean \pm standard deviation or number (\%). *Antiarrhythmic drugs included class Ic and class III drugs. Osteoporosis medications included bisphosphonates, calcitonin, estrogen preparations, hormone replacement therapy, selective estrogen-receptor modulators, ipriflavone, and recombinant parathyroid hormone. ¥Sedatives included benzodiazepines, chloral hydrate, buspirone, and zopliclone. ACEI, angiotensin-converting enzyme inhibitor; $\mathrm{AF}$, atrial fibrillation; ARB, angiotensin-receptor blocker; BMI, body mass index; CKD, chronic kidney disease; COPD, chronic obstructive pulmonary disease; DM, diabetes mellitus; HF, heart failure; HTN, hypertension; MI, myocardial infarction; PAD, peripheral artery disease; TIA, transient ischemic attack.

vehicle accident on the same date, open fractures, skull/ facial bone fractures, finger fractures, and toe fractures were excluded. Major fracture events were defined as hip fractures, vertebral fractures, and fractures of the pelvis and acetabulum. All remaining bony fractures were considered as minor. The follow-up period was from the index date to the date of either the first fracture, detection of a malignancy, withdrawal from the NHIS-NSC (including death), motor vehicle accident, or the end of data collection (31 December 2013), whichever occurred first. To evaluate deaths after fracture, patients were followed from the time of the first fracture until death during any subsequent inpatient course.

\section{Diagnosis Validation}

In order to evaluate the accuracy of our definition of osteoporosis, we conducted a validation study using medical records between 2009 and 2013. After excluding patients with M82.0 (as described), we identified 5,314 patients using the ICD-10 codes M80-, M81-, and M82-. Osteoporosis diagnosis was based on the patient's BMD, measured using central dual X-ray absorptiometry according to the World Health Organization criteria. ${ }^{22}$ The positive predictive value (PPV) was 96\% (5,101 of 5,314 patients). 


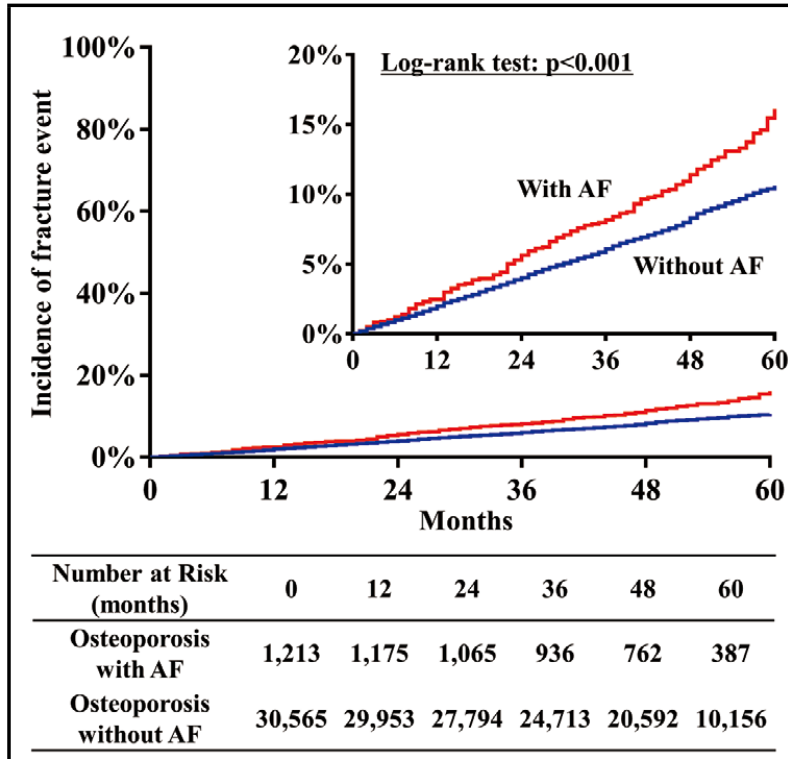

Figure 2. Cumulative incidence of bone fractures according to comorbid atrial fibrillation (AF).

In order to validate our diagnosis of bone fracture, 498 patients were randomly chosen based on their inpatient principal diagnoses using ICD-10 codes from a single tertiary medical center. Their radiographic findings, including $\mathrm{x}$-rays and CT scans, were reviewed. The PPV was $98 \%$ (494 of 504 patients).

The diagnostic accuracy of AF (with a PPV of 94.1\%), MI (with a PPV of $86.5 \%$ ), ischemic stroke (with a PPV of $88-95 \%$ ), and intracranial hemorrhage (with a PPV of $78-92 \%$ ) in the Korean NHIS has been validated previously. ${ }^{19-21,23}$

\section{Propensity Score-Matching Analysis}

We performed propensity score-matching to reduce the potential selection bias associated with an observational study. Propensity scores were estimated using a nonparsimonious multiple logistic regression model. The following 16 variables were entered: age, sex, BMI (body mass index), HF, HTN, DM, previous stroke/TIA, previous MI, PAD, COPD, CKD, CHADS 2 score, $\mathrm{CHA}_{2} \mathrm{DS}_{2}-\mathrm{VASc}$ score, smoking history, alcohol (glasses per week), and physical activity (exercise sessions/week). The cases were then matched (without replacement) with controls 1:1 based on the closest possible value of the propensity score (nearest neighbor matching). The caliper size was set at 0.10 -fold the standard deviation of the logit of the propensity scores. ${ }^{24}$ Based on this propensity score-matching, we assembled a cohort of patients with AF $(n=1,209)$ and matched patients without AF $(n=1,209)$. The balance between the 2 groups was checked by paired comparison tests and standardized differences of the 16 baseline covariates. ${ }^{25}$

\section{Statistical Analysis}

Continuous variables are expressed as mean \pm standard deviation. Normally distributed continuous data in both cohorts were compared using unpaired Student's t-test. Categorical variables were compared with $X^{2}$-analysis with Fischer's exact correction. Kaplan-Meier survival curves for bone fractures and deaths after fractures were constructed. They were compared in-between using the log-rank test. In order to investigate the association between the presence of $\mathrm{AF}$ and the risk of fracture in patients with osteoporosis, we used Cox proportional hazards regression models. We also conducted a sensitivity analysis: to control for possible confounding by instability caused by stroke, the regression models were reevaluated with exclusion of patients who had a stroke history at the time of entry into the cohort.

$\mathrm{P}<0.05$ were considered statistically significant. All analyses were performed using SPSS software version 23.0 (SPSS, Chicago, IL, USA).

\section{Results}

\section{Patients' Characteristics}

The patients' clinical characteristics are presented in Table 1. In general, the AF group was older (69.9 18.4 vs. $65.3 \pm 8.7$, $\mathrm{P}<0.001)$ and included fewer females $(85.0 \%$ vs. $91.9 \%$, $\mathrm{P}<0.001)$ than the non-AF group. The proportions of comorbidities, including HF, HTN, DM, stroke/TIA, MI, $\mathrm{PAD}, \mathrm{COPD}$, and $\mathrm{CKD}$, were higher in the AF group than in the non-AF group (all $\mathrm{P}<0.001$ ). The $\mathrm{CHADS}_{2}$ and $\mathrm{CHA}_{2} \mathrm{DS}_{2}-\mathrm{VASc}$ scores were higher in the AF group (both $\mathrm{P}<0.001)$. The patients in the AF group had higher BMI $(\mathrm{P}<0.001)$ and were less physically active $(\mathrm{P}=0.009)$.

\section{Risk of Osteoporotic Fracture by the Presence of AF}

During a median 48 months of follow-up (interquartile range, 36-60 months), the incidence rates of fracture were higher in the AF group than in the non-AF group (total fracture: 3.20 vs. 2.18 per 100 person-years; major fracture: 1.92 vs. 1.13 per 100 person-years; Table S2). The test for subgroup difference indicated consistent higher fracture risk of the AF group across prespecified subgroups according to age ( $<65$ years; $65-74$ years; $\geq 75$ years) and sex ( $\mathrm{P}$ for interaction, age: 0.206 ; sex: 0.624$)$. The cumulative incidence of fracture events according to the presence of AF is presented in Figure 2. The cumulative incidence of fractures was significantly higher in the AF group than in the non-AF group (log-rank $\mathrm{P}<0.001)$.

$\mathrm{AF}$ was associated with osteoporotic fracture with an unadjusted hazard ratio (HR) of 1.47 (95\% confidence interval [CI], 1.24-1.74; $\mathrm{P}<0.001)$. HRs for the association between $\mathrm{AF}$ and fracture with sequentially greater degrees of multivariable adjustment are shown in Figure 3. After adjusting for age and sex (model 1), the risk of fracture in patients with AF was $23 \%$ higher than in patients without $\mathrm{AF}(95 \% \mathrm{CI}, 446 \% ; \mathrm{P}=0.018)$. With progressive sequential adjustment by comorbidities including HF, HTN, DM, previous stroke/TIA, previous MI, PAD, COPD, and CKD, AF was independently correlated with fracture incidence with a HR of $1.22(95 \% \mathrm{CI}, 1.03-1.45 ; \mathrm{P}=0.021)$ (model 2$)$. In the final fully adjusted model (model 3 ), adjusted for lifestyle including smoking, alcohol intake, and physical activity, and medication use in addition to model 2, the association remained statistically significant, with a $\mathrm{HR}$ of 1.21 (95\% CI, 1.02-1.41; $\mathrm{P}=0.031)$.

The following variables were found to be associated with fractures using multivariate Cox regression analysis of demographic and comorbid variables: older age, female sex, decreased physical activity, DM, stroke/TIA, MI, PAD, loop diuretics use, corticosteroid use, and sedative use (Table S3). In subgroup analyses stratified by sex (Table S4), the association between AF and fracture was 
Model 1 : Adjusted for age and sex

Reference

(without AF)

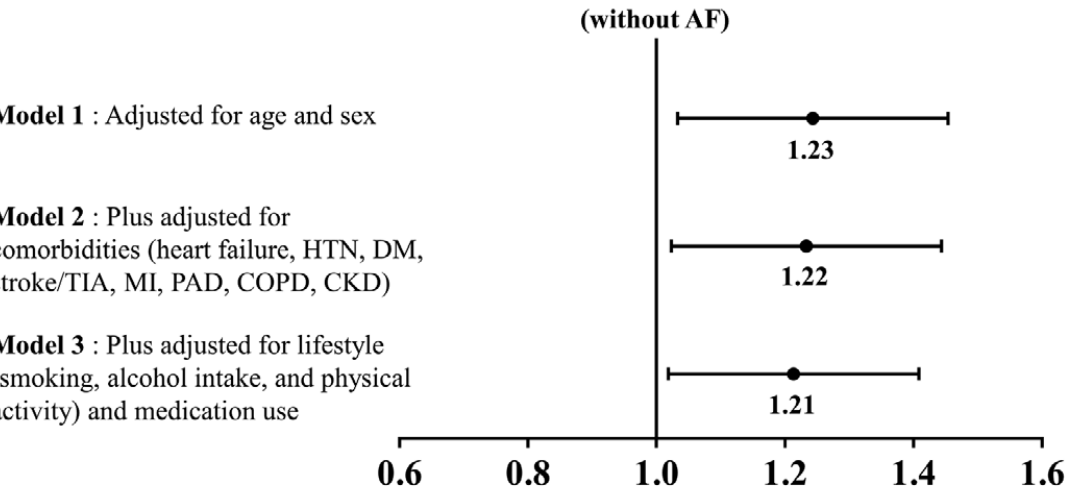

Hazard ratio $(95 \%$ CI $)$

Figure 3. Hazard ratios for the association between atrial fibrillation (AF) and fractures with sequentially greater degrees of multivariable adjustment. $\mathrm{Cl}$, confidence interval; CKD, chronic kidney disease; COPD, chronic obstructive pulmonary disease; DM, diabetes mellitus; HTN, hypertension; $\mathrm{MI}$ myocardial infarction; PAD, peripheral artery disease; TIA, transient ischemic attack. consistently significant in both sexes. In females, increasing alcohol use, smoking, and anticonvulsant use were additionally found to be independently correlated to fracture.

In a sensitivity analysis that excluded patients with stroke history at the time of entry into the cohort $(n=28,944)$, the adjusted risk of fracture had a similar magnitude and was statistically significant (HR, 1.20; 95\% CI, 1.01-1.42; $\mathrm{P}=0.040$ ) in the final fully adjusted model.

Death After Fracture Event According to the Presence of AF The Kaplan-Meier survival curves of patients after fracture according to the presence of AF are presented in Figure 4. During the follow-up after fracture event, $18(12.7 \%)$ patients with AF and $95(3.8 \%)$ patients without AF died (mortality rate, with AF: 6.82 vs. without AF: 1.85 per 100 person-years; log-rank $\mathrm{P}<0.001$ ). The clinical characteristics of the patients with fracture events are presented in Table S5.

Among patients with bone fractures, those with AF had a higher mortality rate than those without AF (adjusted HR, 1.92; 95\% CI, 1.35-3.27; $\mathrm{P}=0.016$ ). Other factors independently associated with death were older age, being underweight (BMI $<18.5 \mathrm{~kg} / \mathrm{m}^{2}$ ), smoking, decreased physical activity, DM, and CKD (Table S6). In the subgroup analyses stratified by sex (Table S7), the association between AF and death was consistently significant in both sexes.

\section{Predictors for Osteoporotic Fracture in Patients With AF}

In patients with AF and osteoporosis, the following variables were identified as predictors of bone fracture using multivariate Cox regression analysis: older age, female sex, being underweight, decreased physical activity (exercising $<3$ times/week), history of stroke/TIA, thiazide use, and sedative use (Table 2 , model 1). Cox regression analysis also demonstrated that higher $\mathrm{CHADS}_{2}(\geq 2$ points) and $\mathrm{CHA}_{2} \mathrm{DS}_{2}-\mathrm{VASc}$ ( $\geq 2$ points) scores independently correlated with the incidence of bone fractures (HR 2.46, 95\% CI 1.62-3.72; P<0.001; and HR 1.61, 95\% CI 1.14$2.82 ; \mathrm{P}=0.007)$, compared with those with scores of $0-1$ point (Table 2, models 2 and 3 ).

\section{Propensity Score-Matching Analysis}

Baseline characteristics after propensity score-matching

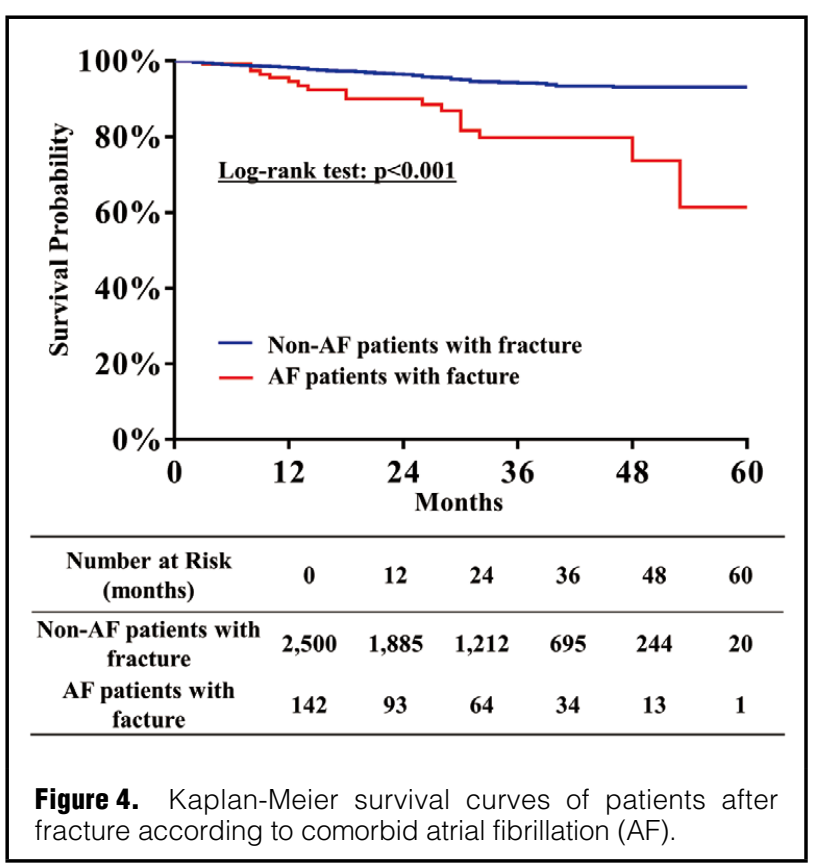

are shown in Table S8. There were no significant differences between the AF and non-AF groups with regard to demographic characteristics and comorbidities.

Similar to the results of the non-matched cohort, the incidence rates of fracture were higher in the AF group than in the non-AF group (total fracture: 3.18 vs. 2.68 per 100 person-years; major fracture: 1.90 vs. 1.54 per 100 person-years; Table S9). AF was associated with a higher risk of fracture in comparison with patients without $\mathrm{AF}$ (HR 1.18, 95\% CI 1.02-1.35; $\mathrm{P}=0.033$ ). Among the patients with bone fractures $(n=259)$, those with AF had a higher mortality rate after the fracture event than did those without AF (6.85 vs. 4.73 per 100 person-years). AF was associated with a higher risk of death after a fracture event (HR 1.44, 95\% CI 1.24-1.69, $\mathrm{P}<0.001$ ). 


\begin{tabular}{|c|c|c|c|}
\hline \multirow{2}{*}{ Variables } & \multirow{2}{*}{ Level } & \multicolumn{2}{|c|}{ Osteoporotic fracture } \\
\hline & & Adjusted HR (95\% Cl) & $P$ value \\
\hline \multicolumn{4}{|l|}{ Model 1a } \\
\hline Age & Per year increase & $1.07(1.05-1.10)$ & $<0.001$ \\
\hline Female & & $2.69(1.33-5.43)$ & 0.006 \\
\hline Underweight & $\mathrm{BMI}<18.5 \mathrm{~kg} / \mathrm{m}^{2}$ & $2.27(1.16-4.47)$ & 0.017 \\
\hline Decreased physical activity & $<3$ times/week of exercise & $1.08(1.01-1.14)$ & 0.020 \\
\hline Ischemic stroke/TIA & & $1.46(1.02-2.09)$ & 0.040 \\
\hline Thiazide use & & $1.35(1.01-1.81)$ & 0.042 \\
\hline Sedative use* & & $1.53(1.14-2.05)$ & 0.020 \\
\hline \multicolumn{4}{|l|}{ Model $2^{b}$} \\
\hline $\mathrm{CHADS}_{2}$ score & $\geq 2$ & $2.46(1.62-3.72)$ & $<0.001$ \\
\hline Female & & $2.52(1.18-5.36)$ & 0.017 \\
\hline Underweight & $\mathrm{BMI}<18.5 \mathrm{~kg} / \mathrm{m}^{2}$ & $2.91(1.49-5.68)$ & 0.002 \\
\hline Sedative use* & & $1.46(1.04-2.07)$ & 0.031 \\
\hline \multicolumn{4}{|l|}{ Model 3c } \\
\hline $\mathrm{CHA}_{2} \mathrm{DS}_{2}$-VASc score & $\geq 2$ & $1.61(1.14-2.82)$ & 0.007 \\
\hline Underweight & $\mathrm{BMI}<18.5 \mathrm{~kg} / \mathrm{m}^{2}$ & $2.91(1.49-5.69)$ & 0.002 \\
\hline Decreased physical activity & $<3$ times/week of exercise & $1.51(1.07-2.14)$ & 0.020 \\
\hline Thiazide use & & $1.52(1.08-2.13)$ & 0.017 \\
\hline Sedative use ${ }^{\star}$ & & $1.57(1.12-2.22)$ & 0.010 \\
\hline
\end{tabular}

aAdjusted for all patient characteristics and medications shown in Table 1. ${ }^{\text {bAdjusted for sex, CHADS} 2 ~ s c o r e, ~}$ BMl-group, lifestyle (smoking, alcohol use, and physical activity), and all medications shown in Table 1. 'Adjusted for $\mathrm{CHA}_{2} \mathrm{DS}_{2}$-VASc score, BMI-group, lifestyle (smoking, alcohol use, and physical activity), and all medications shown in Table 1. *Sedatives included benzodiazepines, chloral hydrate, buspirone, and zopliclone. Cl, confidence interval; HR, hazard ratio. Other abbreviations as in Table 1.

\section{Discussion}

\section{Main Findings}

Using a nationwide cohort of Korean patients with osteoporosis, we found that the incidence of bone fractures was significantly higher in patients with AF than in those without AF. In addition, AF was associated with a $21 \%$ increase in the risk of fractures during a median 4 years of follow-up. This association appeared to remain significant after adjusting for potential confounders such as age, sex, comorbidities, lifestyle, and medication use. The mortality rate after fracture was significantly higher in patients with AF than in those without. After propensity score-matching, AF was consistently associated with a higher risk of osteoporotic fracture and subsequent death after fracture. In AF patients, the risk of fracture was significantly increased in those who were older, female, underweight, and less physically active, with a history of stroke/TIA, thiazide/sedative use, and higher $\mathrm{CHADS}_{2}$ or $\mathrm{CHA}_{2} \mathrm{DS}_{2}-$ VASc score.

\section{Risk of Osteoporotic Fracture and Death After Fracture Event by the Presence of AF}

Our data supported the hypothesis that AF is an independent risk factor for bone fractures. There are several probable explanations for this association. Previous studies have indicated that bone fracture is associated with multiple exogenous and endogenous factors that increase fall risk and weaken bony structures. ${ }^{26-28}$ Sanders et al found that $\mathrm{AF}$ is independently associated with increased falls.10 Possible explanations for this association include hemodynamic alterations with regard to decreased cardiac output from loss of the atrial kick and irregular ventricular response; these changes can impair brain perfusion, causing a loss of postural tone, which in turn, can result in falls. ${ }^{29}$ Patients with AF often have sinus node diseases, ${ }^{30}$ which can result in bradycardia, and even asystole post-AF termination. With regard to bone strength, AF might affect the microvasculature in bones through thromboembolism, thus affecting bone formation. ${ }^{31}$

Recent studies from Taiwan and Australia showed that individuals with AF have a 2-fold increase in risk of fracture, ${ }^{15,17}$ but Wallace et al found no significant association between $\mathrm{AF}$ and fracture risk. ${ }^{16}$ In our study, the association was modest but remained statistically significant, with a $21 \%$ increase in risk. The strength of this study over previous studies is the use of extensive data to adjust for potential confounders that would not have been available in the other studies, including more varied comorbidities, BMI, lifestyle factors, and cardiac/noncardiac medications. It is a novel finding that $\mathrm{AF}$ was associated with a 1.92-fold increase in death among patients after fracture events. Our findings on higher fracture incidence and mortality rates suggested that individuals with $\mathrm{AF}$ may potentially benefit from close screening by clinical history and BMD testing and should be at least carefully monitored after fracture.

\section{Predictors for Osteoporotic Fracture in Patients With AF}

In the general population, older age, low BMI, and decreased physical activity are important risk factors for both osteoporosis and fractures. ${ }^{32,33} \mathrm{We}$ demonstrated that older age, low BMI, and decreased physical activity are independent risk factors for osteoporosis-related fractures 
in AF patients. In our study, although sedative use was associated with increased fracture risk in both the general osteoporosis group and the AF subgroup, thiazide use correlated with fracture only in the AF subgroup. Those medications might be related to increased risk of fall, and the finding implies that thiazide diuretics should be prescribed more carefully in AF patients with osteoporosis. The $\mathrm{CHADS}_{2}$ and $\mathrm{CHA}_{2} \mathrm{DS}_{2}-\mathrm{VASc}$ scores, which are validated tools that predict stroke and systemic embolism in patients with $\mathrm{AF},{ }^{\mathbf{3 4}, 35}$ share common etiological factors with osteoporosis including older age, HF, HTN, and DM. We found that in AF patients, the risk of bone fracture increases with higher $\mathrm{CHADS}_{2}$ or $\mathrm{CHA}_{2} \mathrm{DS}_{2}-\mathrm{VASc}$ score. That finding suggested that among patients diagnosed with AF, the risk of fracture can be detected using a well-known stroke risk score and its cutoff. In doing so, we can identify patients who need to be screened with BMD testing. Furthermore, we can properly counsel these patients with regard to calcium and vitamin D supplementation, regular exercise, smoking cessation, and identification of unnecessary medications.

\section{Antithrombotic Therapy and the Risk of Bone Fractures in Patients With AF}

There is controversy regarding warfarin's deleterious effects on bone density and subsequent increased fracture risk. Several studies have reported that warfarin therapy might be associated with reduced $\mathrm{BMD},{ }^{12,13}$ but this observation is inconsistent across the literature. ${ }^{36}$ Prior groups have investigated whether warfarin exposure is associated with fracture, and have found conflicting results. Gage et al found that long-term $(\geq 1$ year) warfarin use was associated with osteoporotic fractures in men with AF. ${ }^{11}$ In contrast, a more recent Taiwanese national cohort study found that warfarin users had significantly lower risk of bone fractures than did non-warfarin users among AF patients with $\mathrm{CHA}_{2} \mathrm{DS}_{2}-\mathrm{VASc}$ score $\geq 1$ point. ${ }^{17}$ In our study, warfarin use did not independently correlate with the incidence of fracture in the entire osteoporosis population or in the AF subgroup. Further understanding of the mechanisms and randomized prospective studies are needed to resolve the ongoing controversy.

\section{Study Limitations}

First, although we adjusted for many potential risk factors of osteoporotic fracture, we did not measure others, including falls, frailty, BMD, calcium/vitamin D status (supplements are available without prescription), and non-vitamin $\mathrm{K}$ antagonist oral anticoagulant prescription. These unmeasured variables may confound the observed association between AF and fracture. Second, studies using administrative databases are potentially susceptible to errors arising from coding inaccuracies, although we applied the definition that we had already validated in previous studies using a Korean NHIS sample cohort. ${ }^{19-21,23}$ Third, we could not distinguish the types of AF (paroxysmal or nonparoxysmal) and patients with atrial flutter were included without being distinguished. A fourth limitation is that we did not include minor bone fractures not requiring hospitalization. Fifth, we analyzed only all-cause death data and it is unclear whether the increased mortality rate observed was directly related to AF. Sixth, our study only enrolled Asian patients, potentially making it difficult to generalize our results to other populations. Finally, because of the observational nature of this study, we could not establish causality, only correlation.

\section{Conclusions}

Patients with AF were at a higher risk of osteoporotic fracture and subsequent death after fracture than were patients without AF in this study. Older age, female sex, being underweight, decreased physical activity, history of stroke or TIA, usage of thiazides or sedatives, and higher $\mathrm{CHADS}_{2}$ or $\mathrm{CHA}_{2} \mathrm{DS}_{2}$-VASc score were associated with fractures in patients with AF. We suggest that a diagnosis of AF portends a substantially increased risk of fractures and thus such patients require close screening, as well as counseling regarding preventive strategies.

\section{Sources of Funding}

This study was supported by a CMB-Yuhan research grant of Yonsei University College of Medicine (6-2015-0173), research grants from the Basic Science Research Program through the National Research Foundation of Korea funded by the Ministry of Education, Science and Technology (NRF-2012R1A2A2A02045367), and a grant from the Korean Healthcare Technology R\&D project funded by the Ministry of Health \& Welfare (HI16C0058, HI15C1200).

\section{Disclosures}

The authors have no conflicts of interest to disclose.

\section{References}

1. Go AS, Hylek EM, Phillips KA, Chang Y, Henault LE, Selby $\mathrm{JV}$, et al. Prevalence of diagnosed atrial fibrillation in adults: National implications for rhythm management and stroke prevention: The AnTicoagulation and Risk Factors in Atrial Fibrillation (ATRIA) Study. JAMA 2001; 285: 2370-2375.

2. Wattigney WA, Mensah GA, Croft JB. Increasing trends in hospitalization for atrial fibrillation in the United States, 1985 through 1999: Implications for primary prevention. Circulation 2003; 108: $711-716$.

3. McFarlane SI, Muniyappa R, Shin JJ, Bahtiyar G, Sowers JR. Osteoporosis and cardiovascular disease: Brittle bones and boned arteries, is there a link? Endocrine 2004; 23: 1-10.

4. Benjamin EJ, Levy D, Vaziri SM, D'Agostino RB, Belanger AJ, Wolf PA. Independent risk factors for atrial fibrillation in a population-based cohort. The Framingham Heart Study. JAMA 1994; 271: 840-844.

5. Adib Hajbaghery M, Abbasinia M. Quality of life of the elderly after hip fracture surgery: A case-control study. J Caring Sci 2013; 2: 53-59.

6. De Laet CE, van Hout BA, Burger H, Weel AE, Hofman A, Pols HA. Incremental cost of medical care after hip fracture and first vertebral fracture: The Rotterdam study. Osteoporos Int 1999; 10: $66-72$

7. Aluoch AO, Jessee R, Habal H, Garcia-Rosell M, Shah R, Reed $\mathrm{G}$, et al. Heart failure as a risk factor for osteoporosis and fractures. Curr Osteoporos Rep 2012; 10: 258-269.

8. Benzinger P, Rapp K, Konig HH, Bleibler F, Globas C, Beyersmann J, et al. Risk of osteoporotic fractures following stroke in older persons. Osteoporos Int 2015; 26: 1341-1349.

9. Sennerby U, Melhus H, Gedeborg R, Byberg L, Garmo H, Ahlbom A, et al. Cardiovascular diseases and risk of hip fracture. JAMA 2009; 302: 1666-1673.

10. Sanders NA, Ganguly JA, Jetter TL, Daccarett M, Wasmund SL, Brignole M, et al. Atrial fibrillation: An independent risk factor for nonaccidental falls in older patients. Pacing Clin Electrophysiol 2012; 35: 973-979.

11. Gage BF, Birman-Deych E, Radford MJ, Nilasena DS, Binder EF. Risk of osteoporotic fracture in elderly patients taking warfarin: Results from the National Registry of Atrial Fibrillation 2. Arch Intern Med 2006; 166: 241-246.

12. Myers KE, Nakamura JM. Maternal and fetal sequelae of anticoagulation during pregnancy. Am J Cardiol 1990; 65: 1410.

13. Caraballo PJ, Gabriel SE, Castro MR, Atkinson EJ, Melton LJ 3rd. Changes in bone density after exposure to oral anticoagulants: A meta-analysis. Osteoporos Int 1999; 9: 441-448.

14. Thompson B, Towler DA. Arterial calcification and bone 
physiology: Role of the bone-vascular axis. Nat Rev Endocrinol 2012; 8: 529-543.

15. Wong CX, Gan SW, Lee SW, Gallagher C, Kinnear NJ, Lau $\mathrm{DH}$, et al. Atrial fibrillation and risk of hip fracture: A populationbased analysis of 113,600 individuals. Int J Cardiol 2017; 243: $229-232$

16. Wallace ER, Siscovick DS, Sitlani CM, Dublin S, Mitchell P, Robbins JA, et al. Incident atrial fibrillation and the risk of fracture in the cardiovascular health study. Osteoporos Int 2017; 28: $719-725$.

17. Lai HC, Chien WC, Chung CH, Lee WL, Wu TJ, Wang KY, et al. Atrial fibrillation, CHA2DS2-VASc score, antithrombotics and risk of non-traffic-, non-cancer-related bone fractures: A population-based cohort study. Eur J Intern Med 2015; 26: $798-$ 806.

18. Lee J, Lee JS, Park SH, Shin SA, Kim K. Cohort profile: The National Health Insurance Service-National Sample Cohort (NHIS-NSC), South Korea. Int J Epidemiol 2017; 46: e15.

19. Kim TH, Yang PS, Kim D, Yu HT, Uhm JS, Kim JY, et al. CHA2DS2-VASc score for identifying truly low-risk atrial fibrillation for stroke: A Korean nationwide cohort study. Stroke 2017; 48: 2984-2990.

20. Lee HY, Yang PS, Kim TH, Uhm JS, Pak HN, Lee MH, et al. Atrial fibrillation and the risk of myocardial infarction: A nationwide propensity-matched study. Sci Rep 2017; 7: 12716.

21. Lee SS, Ae Kong K, Kim D, Lim YM, Yang PS, Yi JE, et al. Clinical implication of an impaired fasting glucose and prehypertension related to new onset atrial fibrillation in a healthy Asian population without underlying disease: A nationwide cohort study in Korea. Eur Heart $J$ 2017; 38: 2599-2607.

22. Kanis JA, Melton LJ 3rd, Christiansen C, Johnston CC, Khaltaev N. The diagnosis of osteoporosis. J Bone Miner Res 1994; 9: 1137-1141.

23. Park TH, Choi JC. Validation of stroke and thrombolytic therapy in Korean National Health Insurance claim data. $J$ Clin Neurol 2016; 12: 42-48.

24. Austin PC. The use of propensity score methods with survival or time-to-event outcomes: Reporting measures of effect similar to those used in randomized experiments. Stat Med 2014; 33: $1242-1258$

25. Austin PC. Balance diagnostics for comparing the distribution of baseline covariates between treatment groups in propensity-score matched samples. Stat Med 2009; 28: 3083-3107.

26. Watts NB, Lewiecki EM, Miller PD, Baim S. National Osteoporosis Foundation 2008 Clinician's Guide to Prevention and Treatment of Osteoporosis and the World Health Organization Fracture Risk Assessment Tool (FRAX): What they mean to the bone densitometrist and bone technologist. J Clin Densitom 2008; 11: 473-477.

27. Kaminska MS, Brodowski J, Karakiewicz B. Fall risk factors in community-dwelling elderly depending on their physical function, cognitive status and symptoms of depression. Int $J$ Environ Res Public Health 2015; 12: 3406-3416.

28. Rice LA, Ousley C, Sosnoff JJ. A systematic review of risk factors associated with accidental falls, outcome measures and interventions to manage fall risk in non-ambulatory adults. Disabil Rehabil 2015; 37: 1697-1705.

29. Naito M, David D, Michelson EL, Schaffenburg M, Dreifus LS The hemodynamic consequences of cardiac arrhythmias: Evalu- ation of the relative roles of abnormal atrioventricular sequencing, irregularity of ventricular rhythm and atrial fibrillation in a canine model. Am Heart J 1983; 106: 284-291.

30. Andersen HR, Nielsen JC, Thomsen PE, Thuesen L, Mortensen PT, Vesterlund T, et al. Long-term follow-up of patients from a randomised trial of atrial versus ventricular pacing for sick-sinus syndrome. Lancet 1997; 350: 1210-1216.

31. Towler DA. Arteriosclerosis, bone biology, and calciotropic hormone signaling: Learning the ABCs of disease in the bonevascular axis. J Am Soc Nephrol 2015; 26: 243-245.

32. Roy DK, O’Neill TW, Finn JD, Lunt M, Silman AJ, Felsenberg $\mathrm{D}$, et al. Determinants of incident vertebral fracture in men and women: Results from the European Prospective Osteoporosis Study (EPOS). Osteoporos Int 2003; 14: 19-26.

33. Cummings SR, Nevitt MC, Browner WS, Stone K, Fox KM, Ensrud KE, et al. Risk factors for hip fracture in white women: Study of Osteoporotic Fractures Research Group. N Engl J Med 1995; 332: 767-773.

34. Gage BF, Waterman AD, Shannon W, Boechler M, Rich MW, Radford MJ. Validation of clinical classification schemes for predicting stroke: Results from the National Registry of Atrial Fibrillation. JAMA 2001; 285: 2864-2870.

35. Olesen JB, Lip GY, Hansen ML, Hansen PR, Tolstrup JS, Lindhardsen J, et al. Validation of risk stratification schemes for predicting stroke and thromboembolism in patients with atrial fibrillation: Nationwide cohort study. BMJ 2011; 342: d124.

36. Jamal SA, Browner WS, Bauer DC, Cummings SR. Warfarin use and risk for osteoporosis in elderly women: Study of Osteoporotic Fractures Research Group. Ann Intern Med 1998; 128: 829-832.

\section{Supplementary Files}

\section{Supplementary File 1}

Table S1. ICD-10 codes of comorbidities

Table S2. Risk of fracture according to comorbid AF in patients with osteoporosis $(n=31,778)$

Table S3. Cox regression analysis of the predictive factors for fractures in patients with osteoporosis $(n=31,778)$

Table S4. Independent correlates of fracture incidence: multivariable Cox proportional hazards model in subgroups of patients with osteoporosis according to sex

Table S5. Characteristics of patients with fracture event $(n=2,642)$

Table S6. Independent correlates of death after fracture event: multivariable Cox proportional hazards model in patients with fracture event $(n=2,642)$

Table S7. Independent correlates of death after fracture event: multivariable Cox proportional hazards model in subgroups of patients with fracture event according to sex $(n=2,642)$

Table S8. Baseline patient characteristics before and after propensity score-matching

Table S9. Risk of fracture according to comorbid AF in patients with osteoporosis after propensity-matching $(n=2,418)$

Please find supplementary file(s);

http://dx.doi.org/10.1253/circj.CJ-17-1179 